\title{
Minimum-action paths for wave-number selection in nonequilibrium systems
}

\author{
Liyan Qiao, ${ }^{1}$ Zhigang Zheng, ${ }^{2, *}$ and M. C. Cross ${ }^{3, \dagger}$ \\ ${ }^{1}$ Department of Physics, Hangzhou Dianzi University, Hangzhou 310018, China \\ ${ }^{2}$ College of Information Science and Engineering, Huaqiao University, Xiamen 361021, China \\ ${ }^{3}$ Department of Physics, California Institute of Technology, Pasadena, California 91125, USA \\ (Received 10 May 2014; revised manuscript received 7 December 2015; published 12 April 2016)
}

\begin{abstract}
The problem of wave-number selections in nonequilibrium pattern-forming systems in the presence of noise is investigated. The minimum-action method is proposed to study the noise-induced transitions between the different spatiotemporal states by generalizing the traditional theory previously applied in low-dimensional dynamical systems. The scheme is shown as an example in the stabilized Kuramoto-Sivashinsky equation. The present method allows us to conveniently find the unique noise selected state, in contrast to previous work using direct simulations of the stochastic partial differential equation, where the constraints of the simulation only allow a narrow band to be determined.
\end{abstract}

DOI: 10.1103/PhysRevE.93.042204

\section{INTRODUCTION}

Spatially periodic patterns such as regular structures of stripes and lattices are ubiquitous in nature and can be found in diverse far-from-equilibrium systems from developmental biology to weather systems (e.g., Rayleigh-Bénard convection in fluids and Turing patterns in chemical systems) [1]. A key issue in these systems is the selection of patterns from many coexisting metastable states with different geometries and wave numbers $[2,3]$. The answer to this fundamental question still remains a mystery even after decades of explorations.

The presence of noise may cause transitions among different states, and a universal mechanism of state (or pattern) selection is the noise-induced transition. In the limit of weak noise, the probability distribution of patterns will be sharply peaked, defining a noise-selected wave number. In an equilibrium system, such as a spatially periodic crystal, the state selected by this mechanism is easy to understand by resorting to the dominant exponential Boltzmann activation term in the fluctuation rate between two stable states $\propto$ $\exp \left(-\Delta / k_{B} T\right)$, with $\Delta$ being the energy barrier between the two states. The prefactor can be determined in simple situations following the work of Kramers in 1940 [4] and Hänggi et al. [5]. However, for driven nonequilibrium systems, even the dominant term in the format of the fluctuation rate between two stable states is not known in general; there is even no potential analogous to the free energy that prescribes the states and the barriers between them. In many cases the nonlinear system is a nonpotential type. Therefore it is significant to develop alternative approaches to explore this issue, especially for spatially continuous nonequilibrium systems.

A possible way to solve this difficulty is to resort to the minimum-action method (MAM) [6-8]. During the last decade, it has been used to calculate the transition paths and rates for nongradient systems, such as field-driven magnetization reversal of macrospins [9] and micromagnetics [10], thermally induced magnetization reversal of a nanomagnet under the influence of spin transfer torque [11],

\footnotetext{
*zgzheng@hqu.edu.cn

†mcc@caltech.edu
}

and expectations dominated by noise-induced excursions from deterministally stable fix points [12]. In this paper we successfully apply this method to study pattern selections in the well-known Kuramoto-Sivashinsky (KS) system [13-15], identifying a unique noise-selected state. Our findings contrast with previous results from direct simulations of the stochastic partial differential equations [16]. The method can be naturally extended to discussions of pattern selections in other spatially extended systems.

The outline of the paper is as follows. We give the general formulation of minimum-action paths in Sec. II. Section III implements the method for the stabilized Kuramoto-Sivashinsky equation. We present detailed results for the transition path for a particular parameter value, as well as results for the noise selected wave number over essentially the whole range of control parameter values for which the periodic states are stable, and compare the results with previous calculations. We conclude in Sec. IV.

\section{GENERAL IDEA OF MINIMUM-ACTION PATHS}

We begin by reviewing the path-integral approach to dynamical systems with noise. Consider a dynamical system $\boldsymbol{x}(t)$ that is governed by the following stochastic differential equation:

$$
\dot{\boldsymbol{x}}=\boldsymbol{v}(\boldsymbol{x}, t)+\boldsymbol{\xi}(t)
$$

where $\boldsymbol{x}$ is a vector of the dynamical variables, $\dot{\boldsymbol{x}} \equiv d \boldsymbol{x} / d t$, and $\boldsymbol{v}(\boldsymbol{x}, t)$ is the deterministic term that determines the motion of the system in the absence of external noise.

We assume first order equations of motion, with no inertial terms; the whole method can be easily extended for systems where such terms are present. The term $\xi(t)$ in Eq. (2.1) describes the noise. For a nonequilibrium system the noise and the dissipation force may come from different interactions and are not necessarily related to each other. The components of the noise vector are taken to be independent random variables with a Gaussian distribution and zero correlation time $\left\langle\xi_{i}(t) \xi_{j}\left(t^{\prime}\right)\right\rangle=D \delta_{i j} \delta\left(t-t^{\prime}\right)$ with $D$ the noise strength. In the theory of activated processes $D$ is assumed small. For an idea of how one might generalize the method to the case of colored noise, see, for example, Refs. [17-19]. 


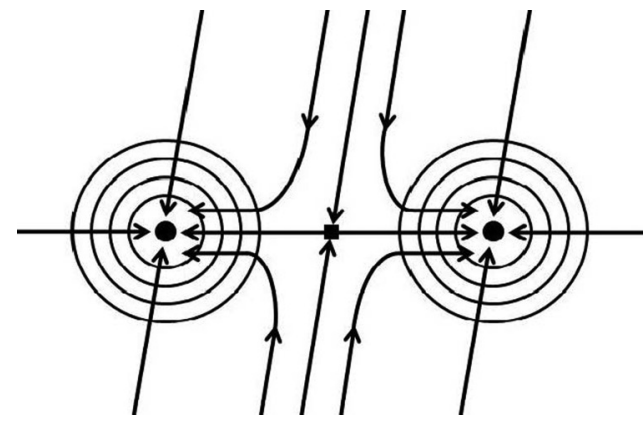

FIG. 1. Cartoon dynamics between the adjacent stable states 1 (denoted by the black dot on the left side) and 2 (denoted by the black dot on the right side). State 3 (denoted by the black square in the middle) is the saddle point between them.

We consider a nonlinear system $\boldsymbol{x}(t)$ with multiple basins of attraction. Stochastic transitions between these different basins of attraction may happen in the presence of noise. Suppose that the deterministic system associated with Eq. (2.1) has two stable equilibrium points, $\boldsymbol{x}_{1}$ and $\boldsymbol{x}_{2}$ in Fig. 1. With the noise, it will be excited from the basin of the stable point $x_{1}$ and eventually make a transition to the vicinity of $\boldsymbol{x}_{2}$ [20]. [21]

The distribution of the noise trajectories $\boldsymbol{\xi}(t)$ is given by

$$
\rho[\boldsymbol{\xi}(t)] \propto \exp \left\{-\frac{1}{4 D} \int \boldsymbol{\xi}(t)^{2} d t\right\} .
$$

Rewriting Eq. (2.1) as $\boldsymbol{\xi}(t)=\dot{\boldsymbol{x}}-\boldsymbol{v}(\boldsymbol{x}, t)$ and introducing the Jacobian $J[x(t)]$ of the variable transformation $\boldsymbol{x} \rightarrow \boldsymbol{\xi}$, we immediately obtain that the probability density for the system to be at $\boldsymbol{x}_{2}$ at time $t$ given that the initial state was at $\boldsymbol{x}_{1}$ at $t=0$ is given by the path-integral formula [22-24]

$$
\rho\left(\boldsymbol{x}_{1}, \boldsymbol{x}_{2}\right) \propto \int_{\text {paths }} J[\boldsymbol{x}(t)] \exp \left[-\frac{S[\boldsymbol{x}(t)]}{4 D}\right] d t,
$$

where the Onsager-Machlup functional $S[x(t)][25,26]$ is

$$
S[x(t)]=\int_{0}^{t} \mathcal{L}(\boldsymbol{x}(t), \dot{\boldsymbol{x}}(t)) d t,
$$

$\boldsymbol{x}_{\tau=0}=\boldsymbol{x}_{1}, \boldsymbol{x}_{\tau=t}=\boldsymbol{x}_{2}$, and the Lagrangian is given by

$$
\mathcal{L}(x(t), \dot{\boldsymbol{x}}(t))=\{\dot{\boldsymbol{x}}(t)-\boldsymbol{v}[\boldsymbol{x}(t)]\}^{2} .
$$

For simplicity, we shall write Eq. (2.3) in the form

$$
\Gamma\left(\boldsymbol{x}_{2}, t \mid \boldsymbol{x}_{1}\right) \propto \Gamma_{0}\left(\boldsymbol{x}_{2}, \boldsymbol{x}_{1}, t\right) \exp \left[-\frac{S[\boldsymbol{x}(t)]}{4 D}\right] .
$$

By analogy with the path-integral formulation from quantum mechanics, we call $S[x(t)]$ the action and $D$ plays the role of $\hbar$ in quantum mechanics. Equation (2.3) is an extension of the Onsager-Machlup result [6] to a multidimensional phase space. A further generalization to multiplicative noise for oneand multi-dimensional systems was done by Graham [24] (for a concise summary, see Sec. III of Graham and Tél [27]).

When $D \ll 1$, this probability density is highly peaked around the path which maximizes $\exp [-S[x(t)] / 4 D]$. According to the variation principle $\delta S[\boldsymbol{x}(t)] / \delta \boldsymbol{x}=0$, the most probable path is the one that minimizes the action in Eq. (2.4) over all the possible trajectories, i.e., the path crosses the saddle point $\boldsymbol{x}_{3}$ on the seperatrix between the basins [21], as is shown in Fig. 1. The saddle point is a stationary solution of the dynamical equations with both stable and unstable eigenvalues, and the geometry is characteristically given by the stable and unstable manifolds. Trajectories that start near or are stochastically placed near the stable manifold of a saddle point will initially move towards the saddle point before moving away along a hyperbolic orbit towards its ultimate attractor in a direction determined by the unstable manifold [28]. The path is denoted as the "optimal path," the path of maximum likelihood, or the "instanton" $\boldsymbol{x}^{*}(t)$, which has the minimum action denoted by $S^{*}$.

The relative escape rates $k_{1 \leftrightharpoons 2}$ between the stable basins of attraction $\boldsymbol{x}_{1}$ and $\boldsymbol{x}_{2}$ are denoted by the forward and backward rates $\Gamma_{\mathbf{1} \rightarrow \mathbf{2}}$ and $\Gamma_{\mathbf{1} \leftarrow \mathbf{2}}$, respectively,

$$
k_{1 \leftrightarrows 2}=\frac{\Gamma_{1 \rightarrow 2}}{\Gamma_{1 \leftarrow 2}}=\frac{\Gamma_{0,1 \rightarrow 2}}{\Gamma_{0,1 \leftarrow 2}} \exp \left[-\left(\frac{\Delta S^{*}[x(t)]}{4 D}\right)\right] .
$$

The prefactor $\Gamma_{0}\left(\boldsymbol{x}_{1}, \boldsymbol{x}_{2}, t\right)$ comes from performing path integrals over variations around the optimal path. This prefactor is generally nonexponential and less sensitive to the parameter values: we will mainly be concerned with the exponential contribution because it will dominate the transition rates in the limit of weak noise.

Since $D \ll 1$, this ratio $k_{1 \leftrightarrows 2}$ is dominated by the exponential factor, and the action difference $\Delta S^{*}[x(t)]=$ $S_{1 \rightarrow 2}^{*}[\boldsymbol{x}(t)]-S_{1 \leftarrow 2}^{*}[\boldsymbol{x}(t)]$ determines the average direction of transitions. Getting the values of $k_{1 \leftrightarrows 2}$ for the various wavenumber states determines which of the many possible stable states is selected by the system.

The above approach has been applied to systems with a few degrees of freedom and described by discrete ordinary differential equations [10], using the Euler-Lagrange equation to give an equation of motion for the optimal path. For systems where the dynamics is derived from a potential, the "uphill" path to the saddle corresponds to the dynamics of the time-reversed deterministic dynamics. The "downhill" path from the saddle simply follows the deterministic dynamics, and does not contribute to the action. (This is shown explicitly for the time-discretized version of our equation in Sec. 1 of Appendix B.) This approach has been extended to continuum systems using direct numerical optimization of the path integral [7,12,29-31]. For transitions between the patterned states with a large number of periods needed to discuss wave-number selection in our continuum system, we could not get the direct optimization method to converge due to the much higher spatial resolution and number of mesh points needed in the discretization. We therefore resorted to an approximate estimate of the optimum path, using the path derived for a potential system (time reversed deterministic path up and deterministic path down) in the spirit of a trial path in a variational approach for our nonpotential system. This approximation is accurate near the threshold of the patterns where nonpotential effects are small, but presumably becomes less accurate away from threshold. 


\section{IMPLEMENTATION OF THE ALGORITHM FOR THE STABILIZED KURAMOTO-SIVASHINSKY EQUATION}

In this study we extend the technique to study transitions between the patterned states of the one-dimensional continuum Kuramoto-Sivashinsky equation, which is described by the following differential equation:

$$
\begin{aligned}
\partial_{t} U(x, t)= & -\alpha U(x, t)-\nabla^{2} U(x, t)-\nabla^{4} U(x, t) \\
& +[\nabla U(x, t)]^{2} .
\end{aligned}
$$

Here, $U(x, t)$ is a dimensionless function (e.g., a front profile) of dimensionless space and time variables $x$ and $t$. The parameter $\alpha$ mimics a stabilizing effect, and $\alpha_{c}$ is the critical value above which the uniform state becomes unstable. We define the reduced parameter $\epsilon$ that measures the distance from the threshold $\epsilon=1-\alpha / \alpha_{c}$ and consider periodic boundary conditions $U\left(x-l_{x} / 2, t\right)=U\left(x+l_{x} / 2, t\right)$ over a domain of length $l_{x}$

A linear stability analysis of Eq. (3.1) about the uniform solution $U(x, t)=0$ with an infinitesimal perturbation $\delta U \sim$ $\exp (\sigma t+i q x)$ (where $q$ is the wave number and $\sigma$ is the amplification rate) gives the dispersion relation $\sigma=-\alpha+$ $q^{2}-q^{4}$. The critical value of $\alpha$ for the onset of the primary instability $\alpha_{c}$ and the bifurcation wave number $q_{c}$ are given by $\alpha_{c}=1 / 4, q_{c}=1 / \sqrt{2}$, which can be obtained from $\sigma=0$ and $\partial \sigma / \partial q=0$

The state $U_{b}=0$ is stable for $\alpha>\alpha_{c}$. For $\alpha<\alpha_{c}$, as $\alpha$ decreases toward zero, this state undergoes a type-Is instability with a critical wave number of $q_{c}$, and spatially periodic steady states emerge [2]. The periodic steady states can themselves become unstable for some parameter values; these instabilities are called secondary instabilities. Misbah and Valance [15] found that besides the usual Eckhaus instability the stabilized KS equation has a variety of other secondary instabilities: (i) period halving of the cellular state, (ii) parity breaking, (iii) vacillating breathing, and (iv) oscillation with a spatial wavelength irrationally related to the basic one. Brunet [32] gives a stability diagram for the $\mathrm{KS}$ equation showing the band of wave numbers $q$ for stable states as a function of $\alpha$.

In the static cells, a band of wave numbers is accessible in Fig. 1 shown in Ref. [32] when the value of $\alpha$ is selected. The system can select one or more discrete values of $q$ within that band. A continuous range of $q$ can be swept out by varying $l_{x}$. There is a discrete set of possible wave numbers in the finite system of length $l_{x}$ :

$$
q_{j}=\frac{2 \pi N_{j}}{l_{x}},
$$

where $N_{j}$ is an integer corresponding to the number of cells in the pattern. We find it convenient to choose a system size

$$
l_{x}=\frac{2 \pi}{q_{c}}\left(N_{c}+1 / 2\right)
$$

where $N_{c}$ is an integer, and set $N_{j}=N_{c}+j$, so the wave numbers $q_{j}$ are given by

$$
q_{j}-q_{c}=\frac{2 \pi}{l_{x}}\left(j-\frac{1}{2}\right) .
$$

Since some level of noise is present in all such systems, one universal mechanism of state selection is noise-induced transition between different possible stable stationary states. When the noise is applied to the system, transitions are induced between two adjacent spatially periodic stable states with a wave-number difference $\delta q=2 \pi / l_{x}$. For small enough noise, this distribution will be sharply peaked, defining a noise-selected wave number.

The noise can select one of the states of the deterministic system and does not induce other states. The relative escape rate Eq. (2.7) will determine the relative probability of finding the system in each state, and, in the weak noise limit, will pick out a preferred unique stationary periodic state. We restrict our attention to the spatially uncorrelated Gaussian white noise. This is incorporated using the Langevin equation associated with Eq. (3.1).

\section{A. Saddle solution}

An important ingredient in calculating the escape rate is the saddle solution. It is useful to investigate this first in the vicinity of the onset of the spatially periodic states, $\alpha \sim \alpha_{c}$, where the evolution of $U(x, t)$ in Eq. (3.1) can be obtained quantitatively in terms of the evolution of complex amplitude $A(\boldsymbol{x}, t)$, which can be introduced as an expansion in small $\epsilon$ :

$$
U(x, t)=\frac{3}{4} \epsilon^{1 / 2}\left[A(X, T) e^{i q_{c} x}+\text { c.c. }\right]+O(\epsilon),
$$

where c.c. denotes the complex conjugate, the $O(\epsilon)$ terms include spatial harmonics of the basic periodic variation at $q_{c}$, and the factor $3 / 4$ gives a convenient normalization, simplifying the form of the derived amplitude equation Eq. (3.6) (see Appendix A for the derivation).

The evolution of the amplitude equation is [33-35]

$$
\tau_{0} \partial_{T} A=\xi_{0}^{2} \partial_{X}^{2} A+A\left(1-|A|^{2}\right),
$$

where $\tau_{0}=4$ is the relaxation time and $\xi_{0}=2 \sqrt{2}$ is a coherence length in Eq. (3.1)

The periodic solutions of Eq. (3.6) are given by solutions of the form (up to an arbitrary phase shift or translation)

$$
A(X)=\sqrt{1-Q^{2}} \exp \left(i Q X / \xi_{0}\right),
$$

where $Q$ is the scaled wave number of the periodic states related to the physical wave number by

$$
q=q_{c}+\epsilon^{1 / 2} Q \xi_{0}^{-1} .
$$

In the finite system of size defined by Eq. (3.3) the values of $Q$ are restricted to

$$
Q_{j}=\frac{2 \pi \xi_{0}}{L}\left(j-\frac{1}{2}\right),
$$

with $L=\epsilon^{1 / 2} l_{x}$ the scaled system size. The periodic solutions to the $\mathrm{KS}$ equation are then

$$
U_{p}(x)=\frac{3}{2} \epsilon^{1 / 2}\left[\sqrt{1-Q_{j}^{2}} \cos \left(q_{j} x\right)\right] .
$$

The saddle solutions within the amplitude equation approximation were calculated by Kramer and Zimmermann [35] with the form (up to an arbitrary translation and phase)

$$
A(X)=\sqrt{2}\left[Q^{\prime}+i \beta \tanh \left(\beta X / \xi_{0}\right)\right] \exp \left(i Q^{\prime} X / \xi_{0}\right),
$$

with $\beta=\left[\frac{1}{2}\left(1-3 Q^{\prime 2}\right)\right]^{\frac{1}{2}}$. For large $|X|$ this asymptotes to a periodic solution of scaled wave number $Q^{\prime}$. In the vicinity 
of $X=0$ there is a reduced magnitude and an enhanced phase variation giving an additional total phase variation of $2 \tan ^{-1}\left(\beta / Q^{\prime}\right)$. Matching the total phase difference across the system to the phase difference for the periodic state with scaled wave number $Q_{j}$ gives the saddle wave number $Q_{j}^{\prime}$ :

$$
Q_{j}=Q_{j}^{\prime}+2 \xi_{0} L^{-1} \tan ^{-1}\left(\beta / Q_{j}^{\prime}\right) .
$$

Note that $\left|Q_{j}\right|>\left|Q_{j}^{\prime}\right|$. This means that

$$
\begin{aligned}
& Q_{j-1}<Q_{j}^{\prime}<Q_{j}, \quad \text { when } j \geqslant 1, \\
& Q_{j}<Q_{j}^{\prime}<Q_{j+1}, \quad \text { when } j \leqslant 0,
\end{aligned}
$$

with $Q_{0}^{\prime}=Q_{1}^{\prime}=0$ labeling the same saddle between the periodic states with scaled wave numbers $Q_{0,1}= \pm \pi \xi_{0} / L$.

The expression for $U(x)$ for the saddle solution corresponding to Eq. (3.11) is

$U_{s}(x)=\frac{3 \sqrt{2}}{2} \epsilon^{1 / 2}\left[Q_{j}^{\prime} \cos \left(q_{j}^{\prime} x\right)-\beta \tanh \left(\beta \epsilon^{1 / 2} x / \xi_{0}\right) \sin \left(q_{j}^{\prime} x\right)\right]$,

with

$$
q_{j}^{\prime}=q_{c}+\epsilon^{1 / 2} Q_{j}^{\prime} \xi_{0}^{-1} .
$$

We now use Eq. (3.14) as the basis of an initial ansatz for the saddle solution in our numerical scheme. However, when the control parameter $\alpha$ is far from the onset $\alpha_{c}$, higher-order terms in Eq. (3.5) are non-negligible. To extend the range of validity we explicitly calculate the $O(\epsilon)$ corrections. This gives the modified saddle solution:

$$
U_{m s}(x)=U_{s}(x)+\left[\frac{3}{2} \sqrt{2} \epsilon^{1 / 2} \beta \tanh \left(\beta \epsilon^{1 / 2} x / \xi_{0}\right)\right]^{2},
$$

with $q_{j}^{\prime}$ given in Eq. (3.15).

Equation (3.16) gives a particular configuration for the saddle solution, which is symmetric about the point of minimum magnitude. Since $U \rightarrow-U$ is not a symmetry of Eq. (3.1) or the saddle solution Eq. (3.16), multiplying $A(X)$ in Eq. (3.11) by $e^{-i \pi}$ and following the same procedure gives another inequivalent, symmetric solution. Multiplying by $e^{-i \pi / 2}$ gives a third solution that is locally antisymmetric about the center. The three saddle solutions are shown in Fig. 2. They were already presented by Kramer and Zimmermann, who demonstrated the existence of these distinct types of saddle-point solutions near threshold and in the fully nonlinear region [35].

\section{B. Numerical results}

Here we first show results for $\alpha=0.225$ to illustrate how our numerical method can be used to determine the preferred wave number. The stable band is limited by the Eckhaus instability at wave numbers $q_{c} \pm \xi_{0}^{-1} \epsilon^{1 / 2} / \sqrt{3}$ [36]. This gives the stable band $0.62<q<0.78(-5<j<6)$.

To determine the preferred wave number between two definite states $q_{0}$ and $q_{1}$, we have to construct a connected path between these two states which goes though some saddle states and compare the actions of the time reversed deterministic paths running from the saddle to the two fixed points considering that there are more than one saddle points between the two metastable states in a general case. After the

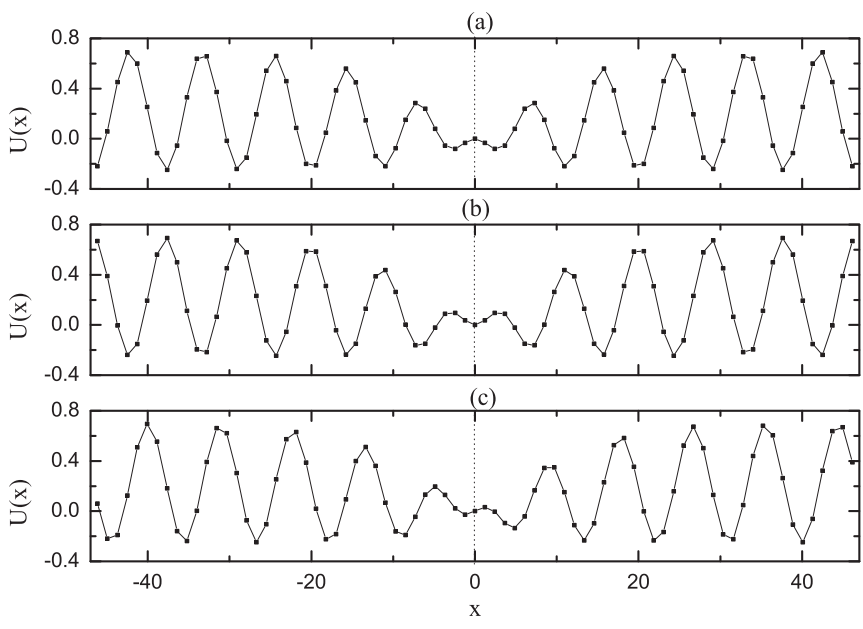

FIG. 2. Localized saddle-point solutions from the amplitude equation. (a) Symmetric case given by Eq. (3.16) with $\mathcal{L} \approx 2.190$, called symmetric-up (SU) from the shape of the center part. (b) Symmetric case given by a $\pi$ phase shift, called symmetric-down (SD) with $\mathcal{L} \approx 2.190$. (c) Antisymmetric case (AS) given by Eq. (3.16) with a $\pi / 2$ phase shift with $\mathcal{L} \approx 2.190$. Parameters used were $\epsilon=0.1$, $l_{x}=123 \sqrt{2} \pi$, and $j=0$.

comparison, we find the most probable escape path with the minimum action.

From Eqs. (2.4) and (2.5), the minimum action corresponding to the KS equation from the spatially periodic stable state $q_{j-1}$ to the adjacent periodic stable state $q_{j}$ is

$$
S_{q_{j-1} \rightarrow q_{j}}^{*}=\int_{0}^{t} \mathcal{L}(t) d t
$$

with $\mathcal{L}(t)=\int_{-l_{x} / 2}^{l_{x} / 2}\left\{\partial_{t} U(x, t)-v[U(x, t)]\right\}^{2} d x$, where $\partial_{t} U$ is given by the evolution according to Eq. (3.1) from the saddle between the two periodic states $j-1, j$ back to the periodic state $j-1$ and $v[U(x, t)]$ is the right hand side of the SKS equation. The value of $q^{\prime}$ characterizing the saddle is defined by Eqs. (3.13) and (3.15).

According to these considerations, we employ the modified saddle solution Eq. (3.16) with various choices of phases to give symmetric-up (SU), symmetric-down (SD), and antisymmetric (AS) initial guesses [35], as initial conditions for numerical refinement to give the final estimate $U_{m s}(x)$ for the saddle state. We refine the solution using the conjugate gradient method to minimize the Lagrangian [37], which is zero at the saddle point. Sometimes we also use the Newton-Raphson method as a better approximation and a preparatory step to get our solution closer to the saddle state before applying the conjugate gradient method.

To determine the evolution of the Lagrangian, we solve Eq. (3.1) numerically with the modified saddle solution Eq. (3.16) as the initial state and employ a finite difference approximation by discretizing the spatial coordinate with a mesh size $\Delta x=l_{x} / n$ for the spatial derivatives and study the time evolution in Mathematica using the method of lines (see Chap. 27 of Ref. [38] for details).

In Figs. 3(a)-3(c), we display the Lagrangian values obtained from Eq. (2.5) for the paths from $q_{0}$ to $q_{1}$ periodic states from three saddle initial guesses with $q_{0}^{\prime}=q_{c}$. By 

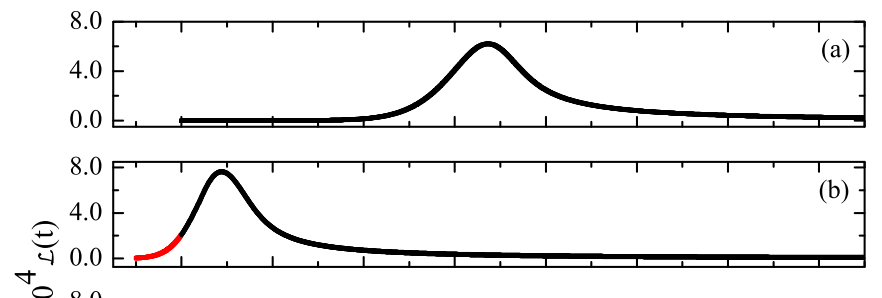

$+$
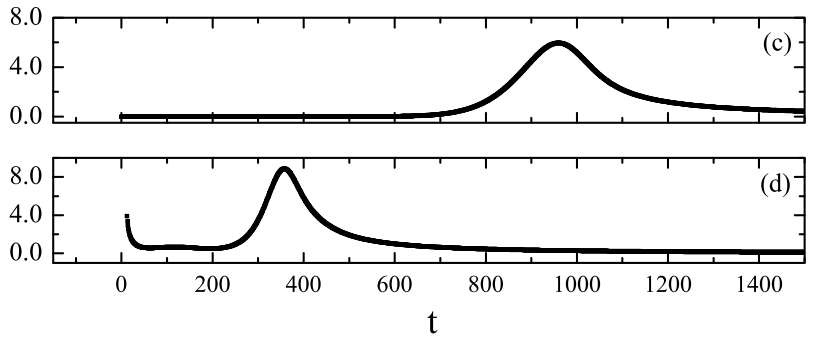

FIG. 3. The Lagrangian as a function of time for the path from $q_{0}$ to $q_{1}$ periodic state. (a) $S_{a}=0.1861$ with SU initial guesses. (b) $S_{b}=$ 0.16946 with SD initial guesses. The red portion of the curve (the part when time is less than zero) in panel (b) shows the extrapolation procedure used to determine the early time behavior. (c) $S_{c}=0.1744$ with AS initial guesses. (d) The Lagrangian as a function of time for the path from $q_{1}$ to $q_{0}$ periodic state corresponding to panel (b). $S_{2}^{*}=0.15937$. The first 12 points are not shown. Parameters used here are $\epsilon=0.1, N_{c}=61, n=450, L=123 \sqrt{2} \pi$.

comparing the actions for those three cases, we can find that the path from saddle (b) has the lowest action $S_{1}^{*}=0.16946$.

The small errors in the numerical estimation for the saddle indicate that the initial condition will lie in the basin of attraction of one of the fixed points. To obtain an initial condition near the saddle and in the basin of attraction of the other fixed point with wave number $q_{0}$, we use $U_{s}^{\prime}(x)$ defined by extrapolating back through the fixed point, $U_{s}^{\prime}(x)=$ $2 U_{s}(x)-U\left(x, t_{1}\right)$, with the moment $t_{1}$ chosen to optimize $U_{s}^{\prime}(x)$. The results for the Lagrangian on the path to the $q_{1}$ fixed point using $t_{1}=70$ are shown in Fig. 3(d). Even after carefully choosing $t_{1}$, the new initial condition still has a significant projection on the stable directions near the saddle (the initial value of the Lagrangian, not shown on the plot, is about 0.02348 ), but it relaxes away over a short evolution time $\sim 100$ : we ignore this portion of the evolution in calculating the action. Thus the action $S_{2}^{*}$ is obtained integrating the curve in Fig. 3(d) from $t=200$ to 1000 , yielding $S_{2}^{*}=0.15937$.

The same method can be used to get the minimum actions for the transitions among other neighboring wave-number pairs $S_{q_{-1} \leftrightarrows q_{0}}^{*}, S_{q_{1} \leftrightarrows q_{2}}^{*}, \ldots$..

The results and the differences $\Delta S^{*}$ are shown in Table I for $\epsilon=0.1$. It is easy to get the interpolated wave number

TABLE I. The minimum actions $S_{j \rightarrow j+1}^{*}$ and $S_{j \leftarrow j+1}^{*}$ and their difference for $\epsilon=0.1$ and $L=123 \sqrt{2} \pi$.

\begin{tabular}{lcccr}
\hline \hline$j$ & $N_{j}$ & $S_{1}^{*}=S_{q_{j} \rightarrow q_{j+1}}^{*}$ & $S_{2}^{*}=S_{q_{j} \leftarrow q_{j+1}}^{*}$ & $\Delta S^{*}=S_{1}^{*}-S_{2}^{*}$ \\
\hline-1 & 60 & 0.08908 & 0.23814 & -0.14906 \\
0 & 61 & 0.16946 & 0.15937 & 0.01009 \\
1 & 62 & 0.25909 & 0.13512 & 0.12397 \\
2 & 63 & 0.34134 & 0.07038 & 0.27096 \\
\hline \hline
\end{tabular}

$q_{s 0}=0.7004$ when $\Delta S=0$. This is the value of $q$ such that the actions for the paths between $q_{s 0}$ and $q_{s 0}+\frac{2 \pi}{l_{x}}$ in the two directions would be equal. The noise preferred wave number is midway between these values, i.e., $q^{*}=0.7062$.

We applied this approach and performed a series of numerical simulations for $\alpha$ over the range $0.145 \leqslant \alpha \leqslant 0.24$, which covers most of the range for which stable periodic states exist. When $\alpha \sim \alpha_{c}$, Eq. (3.6) is an accurate approximation and provides an alternative approach for obtaining the noise selected wave number. Since the amplitude equation is derived from a potential, in this limit the noise selected state is the one that minimizes the potential, which is simply the critical wave number $q_{c}$. Higher-order corrections to the amplitude equation would give the order $O(\epsilon)$ corrections to this result. Thus small $\epsilon$ values of $q^{*}$ should extrapolate to $q_{c}$, providing a check on the numerical results. The lower limit of $\alpha$ investigated is set by the proximity to the oscillatory instability boundary. For the results further away from the onset (e.g., $\alpha<0.16$ ), where the spatial extent of the reduced amplitude region of the saddle state is reduced, we used a smaller system size $l_{x}=61 \sqrt{\pi}$ corresponding to $N_{c}=30$.

\section{Discussion of results}

The results we have displayed so far have been obtained from finite-difference numerical calculations with finite mesh size and may lead to discretization errors in the estimation of the critical wave number at the onset. We therefore need a way of extrapolating the results to larger $n$. To do this extrapolation, we use the expression (see Appendix B for details)

$$
q_{\Delta x \rightarrow 0}^{*}=\frac{2}{\Delta x} \sin \left(\frac{q^{*} \Delta x}{2}\right),
$$

with $\Delta x$ the mesh size, which comes from Eq. (B8) for a periodic solution. Substituting the numerical values of $q^{*}$ and $\Delta x$ in the right hand side, we obtain $q_{\Delta x \rightarrow 0}^{*}$ as the estimate value of the selected wave number with $\Delta x \rightarrow 0$. The results for this extrapolated selected wave number $q_{\Delta x \rightarrow 0}^{*}$ are shown in Fig. 4. Except for the two values of $\alpha$ closest to the onset, we get a rather consistent value of $q^{*}$ extrapolated to zero mesh size for the different meshes used. The differences close to the onset probably arise because the Lagrangian becomes very small here from $\mathcal{L} \sim \epsilon^{3}$, and the numerical extraction of the difference in the actions becomes hard.

We summarize the results of Fig. 4 into the stability diagram of the primary and secondary states from Ref. [32] and obtain Fig. 5. The solid line with crosses is the average noise selected wave number $\overline{q_{\Delta x \rightarrow 0}^{*}}$ found from the different mesh sizes in Fig. 4, with the red error bars showing an overestimate as they represent the maximum possible uncertainty. Note that the two points at the smallest values of $\alpha$ are obtained from results at a single mesh size, and so there are no error bars. The dashed lines with diamonds represent that the Eckhaus band of spatially periodic stable states shrinks in the presence of noise, as shown by Obeid et al. [16], using direct simulations over long times of the noisy Kuramoto-Sivashinsky equation. However they were unable to find the uniquely selected state except close to the onset in the low noise limit for most parameter values due to limitations in the duration of the numerical simulations that were feasible. The path-integral 


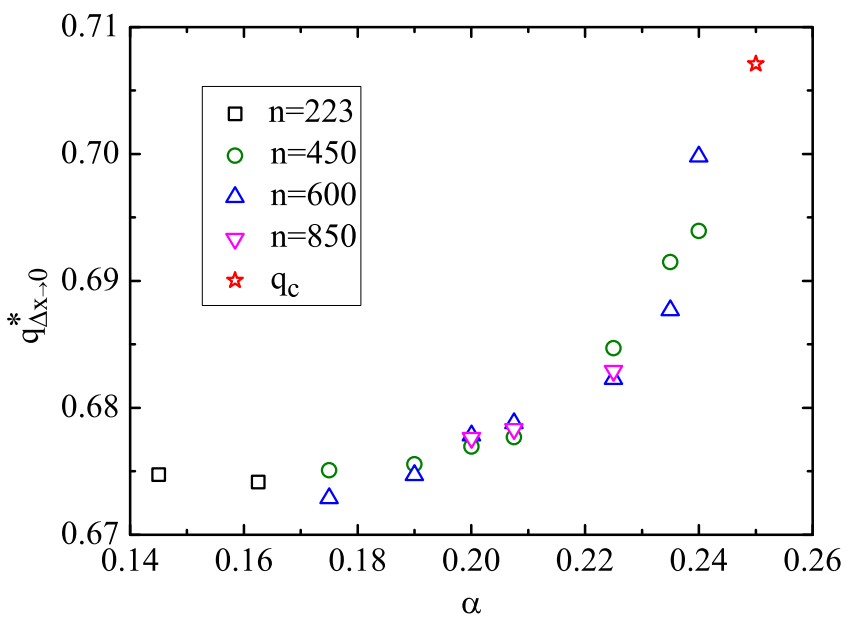

FIG. 4. $q^{*}$ extrapolated to zero mesh size $q_{\Delta x \rightarrow 0}^{*}$ as a function of the parameter $\alpha$. Different symbols correspond to different number of mesh points listed in the legend. The system size was $l_{x}=123 \sqrt{2} \pi$ except for $n=223$ points where $l_{x}=61 \sqrt{2} \pi$. The selected wave number for $\alpha \rightarrow 0.25$ is $q_{c}$ shown by the star.

method is much more reliable here: it does not rely on numerical simulation parameters, such as the mesh size or the evolution time. Moreover, we do not need to limit the control parameter to $0.16 \leqslant \alpha \leqslant 0.25[15,16,32]$. Our method is feasible in the static cells until the band of the selected wave number is getting closer to the oscillating cell (OSC) regime, where it is hard to distinguish the stable states.

\section{CONCLUDING REMARKS}

In conclusion, in this paper we have presented an extensive study on the problem of wave-number selection in

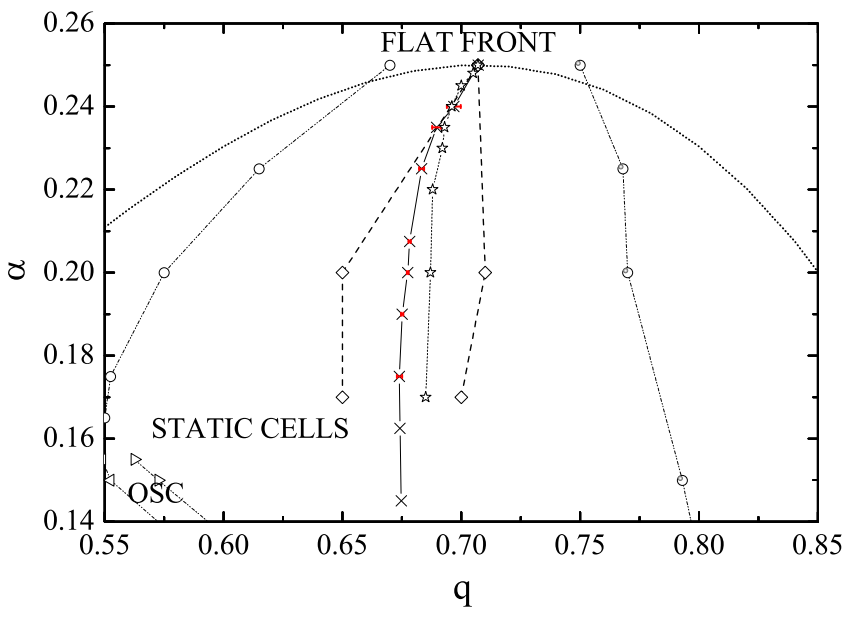

FIG. 5. Noise selected wave numbers. Cross and red error bars with solid line denote results from our calculation. Diamonds with dashes and dots denote results from the stochastic simulations of Obeid et al. [16] showing the reduced band of spatially periodic states in the presence of the noise. Stars with dots denote the wave number maximizing the phase-diffusion coefficient, computed in Ref. [16]. Open circles with dashes denote stability boundaries taken from the calculations of Brunet [32]. nonequilibrium spatiotemporal systems. The path-integral method previously applied to low dimensional systems is developed to apply for spatiotemporal systems described by the partial differential equation. We take the stabilized Kuramoto-Sivashinsky equation by noise induced fluctuations as an example to present how the method works. We have measured the relative transition rates between the stable states based on the search for the optimal transition path and obtain the band of the preferred wave number. It allows us to find the unique noise selected state in the static cells. These numerical results are complemented by a treatment of the problem near the threshold of pattern formation, where the amplitude equation derived using multiple scales perturbation methods holds. Together, these results provide the selected wave number over essentially the full range of stable periodic states, allowing us to test hypotheses for the selected wave number. In addition, our results provide numerous interesting insights into the dynamics of the state selection transitions. The methods we developed here can be extensively applied to a variety of other problems in spatial continuum or discrete network systems, such as state selection in networks of neurons, or collective noise-induced phase slips in large arrays of synchronized oscillators.

\section{ACKNOWLEDGMENTS}

This work has been financially supported by grants from the National Natural Science Foundation of China (Grant No. 11475022) and the Scientific Research Fund of Huaqiao University. The support provided by China Scholarship Council under Grant No. 2010604108 during a visit of L.Q. to Caltech is acknowledged.

\section{APPENDIX A: DERIVATION AND SCALING OF THE AMPLITUDE EQUATION}

Here we derive the amplitude equation for the stabilized Kuramoto-Sivashinsky equation by the method of the multiple scales perturbation theory.

The uniform base state independent of spatial variables is $U_{b}=0$. An arbitrary perturbation $U_{p}(x, t)=U(x, t)-$ $U_{b}(x, t)$ will also satisfy the SKS equation Eq. (3.1). Then we get a nonlinear partial differential equation of the form

$$
\partial_{t} U_{p}=\hat{L} U_{p}+\hat{N}\left[U_{p}\right] .
$$

Here $\hat{L}$ is a linear differential operator that depends on the control parameter and $\hat{N}$ is an operator that collects all the terms that are nonlinear in $U_{p}$.

A primary Turing bifurcation occurs at a critical value $\alpha_{c}=$ $1 / 4$ and $q_{c}=1 / \sqrt{2}$. We expand the linear operator $\hat{L}$ and $U_{p}(x, t)$ in powers of $\epsilon^{1 / 2}$ :

$$
\begin{aligned}
& \hat{L}=\epsilon^{1 / 2} \hat{L}_{0}+\epsilon \hat{L}_{1}+\epsilon^{3 / 2} \hat{L}_{2}+\cdots, \\
U_{p}(x, t)= & U(x, t)-U_{b}(x, t) \\
= & \epsilon^{1 / 2} U_{0}(x, t)+\epsilon U_{1}(x, t)+\epsilon^{3 / 2} U_{2}(x . t)+\cdots,
\end{aligned}
$$


with $U_{0}(x, t)$ given as some slowly varying amplitude $A_{0}(X, T)$ of the critical mode:

$$
U_{0}(x, t)=A_{0}(X, T) e^{i q x}+\text { c.c. }
$$

Here $X$ and $T$ are the slow space and time scales:

$$
\begin{aligned}
& X=\epsilon^{1 / 2} x, \\
& T=\epsilon t .
\end{aligned}
$$

Now collect terms at each order in $\epsilon^{1 / 2}$ in the expansion of Eq. (3.1). At $O\left(\epsilon^{1 / 2}\right)$, we find

$$
\hat{L_{0}} U_{0}=0 \text {, }
$$

where $\hat{L_{0}}=-\partial_{x}^{2}-\partial_{x}^{4}-1 / 4$ and we could get the value for $q=1 / \sqrt{2}=q_{c}$ in Eq. (A4)

At $O(\epsilon)$, we get

$$
\hat{L_{0}} U_{1}+\hat{L_{1}} U_{0}=0,
$$

where $\hat{L_{1}}=-2 \partial_{x} \partial_{X}-4 \partial_{x}^{3} \partial_{X}$.

Equation (A7) can be solved to give

$$
\begin{aligned}
U_{1}(x, t)= & A_{1}(X, T) e^{i x / \sqrt{2}}+\bar{A}_{1}(X, T) e^{-i x / \sqrt{2}} \\
& -\frac{2}{9}\left(A_{0}^{2}(X, T) e^{i x \sqrt{2}}+\bar{A}_{0}^{2}[X, T) e^{-i x \sqrt{2}}\right] \\
& +4\left|A_{0}(X, T)\right|^{2},
\end{aligned}
$$

which introduces the next-order correction $A_{1}(X, T)$ to the amplitude. Here $\overline{A_{0}}(X, T)$ and $\overline{A_{1}}(X, T)$ indicate the complex conjugates of $A_{1}(X, T)$ and $A_{1}(X, T)$, respectively.

At $O\left(\epsilon^{3 / 2}\right)$, we have the equation

$$
\hat{L_{2}} U_{0}=\partial_{T} U_{0}-\hat{L_{0}} U_{2}-\hat{L_{1}} U_{1}-\hat{N}_{1}\left(U_{0}, U_{1}\right),
$$

where $\quad \hat{L}_{2}=-\partial_{X}^{2}-6 \partial_{x}^{2} \partial_{X}^{2}+\frac{1}{4} \quad$ and $\quad \hat{N}_{1}\left(U_{0}, U_{1}\right)=$ $2 \partial_{x} U_{0} \partial_{X} U_{1}+\partial_{x} U_{0} \partial_{X} U_{0}$.

Evaluating all the terms on the right hand side gives

$$
\begin{aligned}
\hat{L_{2}} U_{0}= & \left(\partial_{T} A_{0}-2 \partial_{X}^{2} A_{0}-\frac{A_{0}}{4}+\frac{4}{9} A_{0}\left|A_{0}\right|^{2}\right) e^{i x / \sqrt{2}} \\
& +\left(-\frac{27}{2} i \sqrt{2} \partial_{X}^{2} A_{0}^{2}-i \sqrt{2} A_{0} \partial_{X} A_{0}+A_{0} A_{1}\right) e^{\sqrt{2} i x} \\
& +\left(-\frac{9}{2} A_{0}^{3}\right) e^{3 i x / \sqrt{2}} \\
& +i \sqrt{2} \bar{A}_{0} \partial_{X} A_{0}-\overline{A_{0}} A_{1}+\text { c.c. }
\end{aligned}
$$

The amplitude equation for $A_{0}$ arises as the solvability condition for this equation. The solvability condition arises because the function $e^{i x / \sqrt{2}}$ satisfies the homogeneous equation

$$
\hat{L_{0}} e^{ \pm i x / \sqrt{2}}=0 \text {. }
$$

Thus the coefficient of the $e^{ \pm i x}$ dependence on the right-hand side of Eq. (A10) must be set to zero. This yields the amplitude equation

$$
\partial_{T} A_{0}=2 \partial_{X}^{2} A_{0}+\frac{1}{4} A_{0}-\frac{4}{9} A_{0}\left|A_{0}\right|^{2} .
$$

Rescaling the amplitude $A_{0}=\frac{3}{4} A_{0}^{\prime}$, we get the canonical form of the amplitude equation

$$
4 \partial_{T} A_{0}^{\prime}=8 \partial_{X}^{2} A_{0}^{\prime}+A_{0}^{\prime}\left(1-\left|A_{0}^{\prime}\right|^{2}\right),
$$

which has the same form as the equation in Ref. [35]:

$$
\tau_{0} \partial_{T} A=\xi_{0}^{2} \partial_{X}^{2} A+A\left(1-|A|^{2}\right),
$$

with the decay time $\tau_{0}=4$ and coherence length $\xi_{0}=2 \sqrt{2}$.

\section{APPENDIX B: DISCRETIZATION}

The evolution of $U(x, t)$ from the saddle is approximated by discretizing the space coordinate: we divide the interval $\left[-l_{x} / 2, l_{x} / 2\right]$ into $n$ subintervals of length $\Delta x=l_{x} / n$ with the points $x_{i}=i \Delta x-l_{x} / 2, i=0,1, \ldots, n$. and solve for $U_{i}(t)=$ $U\left(x_{i}, t\right)$.

To derive differential equations for $U_{i}(t)$ we approximate the spatial derivatives $\nabla U, \nabla^{2} U$, and $\nabla^{4} U$ by finite differences and combine them with Eq. (3.1) to obtain the spacediscretized differential equation for the time derivatives $U_{i}^{\prime}(t)$ :

$$
\begin{aligned}
& U_{i}^{\prime}(t)+\alpha U_{i}(t)+\frac{U_{i-1}(t)-2 U_{i}(t)+U_{i+1}(t)}{(\Delta x)^{2}} \\
& +\frac{U_{i-2}(t)-4 U_{i-1}(t)+6 U_{i}(t)-4 U_{i+1}(t)+U_{i+2}(t)}{(\Delta x)^{4}} \\
& -\left(\frac{U_{i+1}(t)-U_{i-1}(t)}{2 \Delta x}\right)^{2}=0, \quad i=0,1, \ldots, n .
\end{aligned}
$$

\section{Transition path}

The fluctuation path minimizes the action with the Lagrangian

$$
\mathcal{L}=\sum_{i}\left\{\dot{U}_{i}(t)-v_{i}\left[U_{k}(t)\right]\right\}^{2},
$$

where $v_{i}$ is the right hand side of the SKS equation with discretized derivatives. Note that $v_{i}$ will depend on $U_{k}$ for sites $k$ in the stencil of the discretization about $i$.

The Euler-Lagrange equation giving the $U_{i}$ minimizing the action is

$$
\frac{d}{d t}\left(\frac{\partial \mathcal{L}}{\partial \dot{U}_{i}}\right)-\frac{\partial \mathcal{L}}{\partial U_{i}}=0 .
$$

Substituting Eq. (B2) into Eq. (B3) yields

$$
\ddot{U}_{i}-\sum_{j} v_{j} \frac{\partial v_{j}}{\partial U_{i}}+\sum_{j} \dot{U}_{j}\left(\frac{\partial v_{j}}{\partial U_{i}}-\frac{\partial v_{i}}{\partial U_{j}}\right)=0 .
$$

For a potential system, the last term of Eq. (B4) disappears, leading to the conclusion that the fluctuation path is the time reversed deterministic path. However, the last term in Eq. (B4) is not zero for the $(\nabla U)^{2}$ term, which is the "nonpotential" term in the SKS equation. This term gives a contribution to $v_{i}$, denoted by $\tilde{v}$ :

$$
\tilde{v}_{i}=\frac{\left(U_{i+1}-U_{i-1}\right)^{2}}{4(\Delta x)^{2}}
$$

which is nonzero in general. The other terms, e.g., $\nabla^{2} U$ and $\nabla^{4} U$, do give zero. Ignoring the last nonpotential term in Eq. (B4), the equation can be integrated to give

$$
\dot{U}_{i}^{2}=v_{i}^{2}
$$

This shows that the minimum-action "uphill" path is the time reversed deterministic dynamics, as in Ref. [17]. Therefore the 
time reversed deterministic path is used as a trial function in the variation, an approximation in nonpotential system.

\section{Discretization errors}

To check the errors due to the spatial discretization for the finite difference scheme, we evaluate the critical onset solutions of the SKS equation in the form of $U(x)=e^{i q n \Delta x}$ to determine how $\alpha_{c}$ and $q_{c}$ vary with $\Delta x$. Setting $U_{i}^{\prime}$ in Eq. (B1) to zero and retaining only the linear terms gives the equation for $\alpha$ at the onset of the periodic solution:

$$
\alpha=q_{0}^{2}-q_{0}^{4},
$$

with

$$
q_{0}^{2}=\frac{2[1-\cos (q \Delta x)]}{(\Delta x)^{2}}=q^{2}\left[\frac{\sin (q \Delta x / 2)}{(q \Delta x / 2)}\right]^{2},
$$

and $\Delta x=2 \pi / q m$ with $m$ the number of mesh points per wavelength. Equation (B7) shows that $\alpha$ is maximized exactly at $\alpha_{c}=1 / 4$ with the value $q_{0}=q_{c}=1 / \sqrt{2}$.

The error in the value of $q$ can be found by expanding the second expression in Eq. (B8):

$$
\frac{q}{q_{0}}=\left[\frac{\sin (q \Delta x / 2)}{(q \Delta x / 2)}\right]^{-1} \simeq 1+\frac{1}{3 !}\left(\frac{q \Delta x}{2}\right)^{2}+\cdots,
$$

so that the fractional error $\delta_{c}$ in $q_{c}$ caused by the discretization is

$$
\delta_{c} \simeq \frac{1}{24}(q \Delta x)^{2} \simeq \frac{\pi^{2}}{6 m^{2}} .
$$

For $m \sim 8$, for example, this gives an error in $q_{c}$ of about $2.5 \%$, while $m \sim 14$ gives the error $0.839 \%$
[1] M. C. Cross and H. Greenside, Pattern Formation and Dynamics in Nonequilibrium Systems (Cambridge University, New York, 2009).

[2] M. C. Cross and P. C. Hohenberg, Rev. Mod. Phys. 65, 851 (1993).

[3] G. Ahlers, D. S. Cannell, M. A. Dominguez-Lerma, and R. Heinrichs, Physica D 23, 202 (1986).

[4] H. Kramers, Physica 7, 284 (1940).

[5] P. Hänggi, P. Talkner, and M. Borkovec, Rev. Mod. Phys. 62, 251 (1990).

[6] L. Onsager and S. Machlup, Phys. Rev. 91, 1505 (1953).

[7] Weinan E, Weiqing Ren, and Eric Vanden-Eijnden, Commun. Pure Appl. Math. 57, 637 (2004).

[8] M. I. Freidlin and A. D. Wentzell, Random Perturbations of Dynamical Systems (Springer, New York, 2012), Vol. 260.

[9] R. V. Kohn, M. G. Reznikoff, and E. Vanden-Eijnden, J. Nonlinear Sci. 15, 223 (2005).

[10] D. Berkov, J. Magn. Magn. Mater. 186, 199 (1998).

[11] G. D. Chaves-O'Flynn, D. L. Stein, A. D. Kent, and E. VandenEijnden, J. Appl. Phys. 109, 07C918 (2011).

[12] T. Grafke, R. Grauer, T. Schäfer, and E. Vanden-Eijnden, Multiscale Model. Simul. 12, 566 (2014).

[13] G. Sivashinsky, Physica D 17, 243 (1985).

[14] Y. Kuramoto and T. Tsuzuki, Prog. Theor. Phys. 55, 356 (1976).

[15] C. Misbah and A. Valance, Phys. Rev. E 49, 166 (1994).

[16] D. Obeid, J. M. Kosterlitz, and B. Sandstede, Phys. Rev. E 81, 066205 (2010).

[17] A. J. Bray and A. J. McKane, Phys. Rev. Lett. 62, 493 (1989).

[18] A. J. McKane, H. C. Luckock, and A. J. Bray, Phys. Rev. A 41, 644 (1990).
[19] A. J. Bray, A. J. McKane, and T. J. Newman, Phys. Rev. A 41, 657 (1990).

[20] M. Heymann and E. Vanden-Eijnden, Phys. Rev. Lett. 100, 140601 (2008).

[21] M. Dykman and M. Krivoglaz, Zh. Eksp. Teor. Fiz. 77, 60 (1979).

[22] R. P. Feynman and A. Hibbs, Quantum Mechanics and Path Integrals (McGraw-Hill, New York, 1965).

[23] R. Graham and T. Riste, Fluctuations, Instabilities and Phase Transitions (Plenum, New York, 1975).

[24] R. Graham, Z. Phys. B 26, 281 (1977).

[25] F. Haake, Springer Tracts Mod. Phys. 66, 98 (1973).

[26] T. Riste, Fluctuations, Instabilities, and Phase Transitions (Plenum, New York, 1975), p. 19.

[27] R. Graham and T. Tél, Phys. Rev. A 31, 1109 (1985).

[28] J. Cushing, B. Dennis, R. Desharnais, and R. Costantino, J. Anim. Ecol. 67, 298 (1998).

[29] H. C. Fogedby and W. Ren, Phys. Rev. E 80, 041116 (2009).

[30] M. Heymann and E. Vanden-Eijnden, Commun. Pure Appl. Math. 61, 1052 (2008).

[31] X. Zhou, W. Ren, E. Weinan et al., J. Chem. Phys. 128, 10411 (2008).

[32] P. Brunet, Phys. Rev. E 76, 017204 (2007).

[33] L. A. Segel, J. Fluid Mech. 38, 203 (1969).

[34] A. C. Newell and J. Whitehead, J. Fluid Mech. 38, 279 (1969).

[35] L. Kramer and W. Zimmermann, Physica D 16, 221 (1985).

[36] M. Lowe and J. P. Gollub, Phys. Rev. Lett. 55, 2575 (1985).

[37] W. H. Press, P. Flannery, S. A. Teukolsky, W. T. Vetterling et al., Numerical Recipes (Cambridge University, Cambridge, England, 1986).

[38] H. Ruskeepää, Mathematica Navigator: Mathematics, Statistics, and Graphics (Academic, New York, 2004), Vol. 1. 